\title{
ESSENTIAL STATE SURFACES FOR KNOTS AND LINKS
}

\author{
MAKOTO OZAWA \\ (Received 9 May 2009; accepted 14 November 2010) \\ Communicated by C. D. Hodgson
}

\begin{abstract}
We study a canonical spanning surface obtained from a knot or link diagram, depending on a given Kauffman state. We give a sufficient condition for the surface to be essential. By using the essential surface, we can deduce the triviality and splittability of a knot or link from its diagrams. This has been done on the extended knot or link class that includes all semiadequate, homogeneous knots and links, and most algebraic knots and links. In order to prove the main theorem, we extend Gabai's Murasugi sum theorem to the case of nonorientable spanning surfaces.
\end{abstract}

2010 Mathematics subject classification: primary 57M25; secondary 57Q35.

Keywords and phrases: state surface, knot diagram, adequate knot, homogeneous knot, Murasugi sum.

\section{Introduction}

In 1930, Frankl and Pontrjagin [9] proved the existence of a Seifert surface for any knot. In 1934, Seifert [28] gave an algorithm for constructing a Seifert surface from a knot diagram. Seifert's algorithm allows us to construct a spanning surface from a knot diagram, depending on a given Kauffman state [15]. In this paper, we give a sufficient condition for the spanning surface to be essential. By using the essential surface, we show that a knot or link is trivial or split if and only if the diagram is trivial or split respectively under our sufficient condition.

Throughout this paper we work in the piecewise linear category. For knot theory, graph theory and 3-manifold theory terminology, we refer to [5, 7, 17].

In Section 2 we define and give examples of state surfaces and state our main results. We prove some lemmas in Section 3, one of which extends Gabai's Murasugi sum theorem [11], and prove our main theorems in Section 4. In Section 5 we list some problems that are of interest for further study. Finally, in Section 6, we summarize some of the recent progress that has been made since the first draft of this paper.

(c) 2012 Australian Mathematical Publishing Association Inc. 1446-7887/2012 \$16.00 


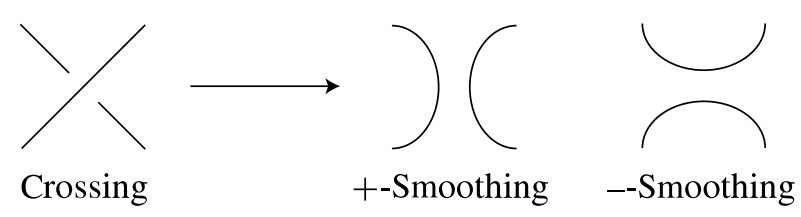

Figure 1. Two smoothings of a crossing.

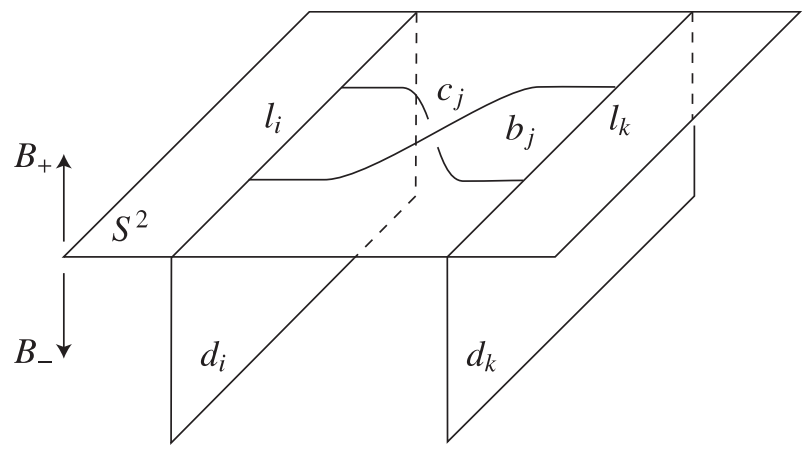

FIGURE 2. Recovering a crossing by a half twisted band.

\section{Definitions, examples and results}

Let $K$ be a knot or link in the 3-sphere $S^{3}$ and let $D$ be a connected diagram of $K$ on the 2-sphere $S^{2}$ that separates $S^{3}$ into two 3-balls, say $B_{+}$and $B_{-}$. Let $C=\left\{c_{1}, \ldots, c_{n}\right\}$ be the set of crossings of $D$. A map $\sigma: C \rightarrow\{+,-\}$ is called a state for $D$. For each crossing $c_{i} \in C$, we take a +-smoothing or --smoothing of $D$, according to whether $\sigma\left(c_{i}\right)=+$ or - (see Figure 1$)$.

Historically, in most papers, +-smoothing and --smoothing are called $A$-splicing and $B$-splicing. The terminology + -smoothing and --smoothing seems reasonable since, if we orient a crossing locally so that it has a \pm -sign, then a smoothing along the orientation coincides with a \pm -smoothing. After \pm -smoothing, we have a collection $l_{1}, \ldots, l_{m}$ of loops on $S^{2}$ that we call state loops. Let $\mathcal{L}_{\sigma}=\left\{l_{1}, \ldots, l_{m}\right\}$ be the set of state loops.

Each state loop $l_{i}$ bounds a unique disk $d_{i}$ in $B_{-}$. We may assume that these disks are mutually disjoint. For each crossing $c_{j}$ and state loops $l_{i}$ and $l_{k}$ whose subarcs replace $c_{j}$ by $\sigma\left(c_{j}\right)$-smoothing, we attach a half twisted band $b_{j}$ to $d_{i}$ and $d_{k}$ so that it recovers $c_{j}$. See Figure 2 for the case where $\sigma\left(c_{j}\right)=+$. In this way, we obtain a spanning surface that consists of disks $d_{1}, \ldots, d_{m}$ and half twisted bands $b_{1}, \ldots, b_{n}$. We call this surface a $\sigma$-state surface $F_{\sigma}$.

ReMark 2.1. The following historical remarks were suggested by Przytycki.

First, the state surfaces corresponding to the positive state $\sigma_{+}$(that is, $\sigma_{+}\left(c_{j}\right)=+$ for all $j$ ) and the negative state $\sigma_{-}$(that is, $\sigma_{-}\left(c_{j}\right)=-$ for all $j$ ) were considered for 
alternating links in the nineteenth century by Tait. They were originally called Tait surfaces, but nowadays are called checkerboard surfaces.

Next, the state surface corresponding to the Seifert state $\vec{\sigma}$ (that is, the state determined by an orientation of the knot) that gives the Seifert surface was introduced by Seifert in [28].

Finally, independently, Przytycki has already considered using a surface for any Kauffman state. See [26, Footnote 2].

We may assume that $F_{\sigma}$ intersects $N(K)$ in its collar $N\left(\partial F_{\sigma} ; F_{\sigma}\right)$ and write $F_{\sigma}$ instead of $F_{\sigma} \cap E(K)$, where $N(K)$ denotes the regular neighbourhood of $K$ in $S^{3}$ and $E(K)$ denotes the exterior of $K$. We take a (twisted) $I$-bundle $F_{\sigma} \tilde{\times} I$ over $F_{\sigma}$ in $E(K)$ and call the associated $\partial I$-bundle $F_{\sigma} \tilde{\times} \partial I$ over $F_{\sigma}$ the interpolating surface obtained from $F_{\sigma}$. We denote this surface by $\left(F_{\sigma}\right)^{\sim}$ since it is a double cover of $F_{\sigma}$. Note that any interpolating surface $\left(F_{\sigma}\right)^{\sim}$ is orientable, and it is connected if and only if $F_{\sigma}$ is nonorientable.

We use $F_{\sigma}$ to construct a graph $G_{\sigma}$, with signs on its edges, by regarding a disk $d_{i}$ as a vertex $v_{i}$ and a band $b_{j}$ as an edge $e_{j}$ that has the same sign as $\sigma\left(c_{j}\right)$. We call the graph $G_{\sigma}$ a $\sigma$-state graph. In general, a graph is called a block if it is connected and has no cut vertex. It is well known that any graph has a unique decomposition into maximal blocks.

Following [4, 16], we say that a diagram $D$ is $\sigma$-adequate if $G_{\sigma}$ has no loops, and that $D$ is $\sigma$-homogeneous if, in each block of $G_{\sigma}$, all edges have the same sign. We remark that any diagram of any link is $\sigma$-adequate for some state $\sigma$ (for example, the Seifert state), and $\sigma^{\prime}$-homogeneous for some state $\sigma^{\prime}$ (for example, the positive state), where the states $\sigma$ and $\sigma^{\prime}$ do not generally coincide.

REMARK 2.2. As was pointed out in [8], the definition of 'adequate' given here seems to differ slightly from the original definition. See Example 2.6 for a discussion of its consistency with the original definition.

Example 2.3. Let $D$ be a diagram of the figure-of-eight knot, which has four crossings, $c_{1}, c_{2}, c_{3}$ and $c_{4}$, as shown at the top of Figure 3. To make a $\sigma$-state surface, let $\sigma\left(c_{1}\right)=\sigma\left(c_{2}\right)=-$ and $\sigma\left(c_{3}\right)=\sigma\left(c_{4}\right)=+$, for example. Since the $\sigma$-state graph $G_{\sigma}$ has no loop and all edges in each block have the same sign as in the second part of Figure 3, $D$ is $\sigma$-adequate and $\sigma$-homogeneous. Moreover, the block decomposition of $G_{\sigma}$ corresponds to a Murasugi decomposition of $F_{\sigma}$ (see the bottom part of Figure 3).

ExAmple 2.4. A diagram $D$ with an orientation is said to be positive if all crossings have a positive sign. For any positive diagram $D$, there exists a state $\sigma$ such that $D$ is $\sigma$-adequate and $\sigma$-homogeneous. Indeed, we can take $\sigma$ so that $\sigma\left(c_{j}\right)=+$ for all $c_{j}$, namely, the positive state $\sigma_{+}$. Also, we can take $\sigma$ so that it yields a canonical Seifert surface $F_{\sigma}$, namely, the Seifert state $\vec{\sigma}$. Note that the states $\sigma_{+}$and $\vec{\sigma}$ coincide only on a positive diagram.

ExAmple 2.5. For any alternating diagram $D$ without nugatory crossings, there exist two states $\sigma_{1}$ and $\sigma_{2}$ such that $D$ is $\sigma_{i}$-adequate and $\sigma_{i}$-homogeneous for $i=1,2$. Indeed, we can take $\sigma_{1}=\sigma_{+}\left(\right.$or $\left.\sigma_{1}=\sigma_{-}\right)$and $\sigma_{2}=\vec{\sigma}$. 


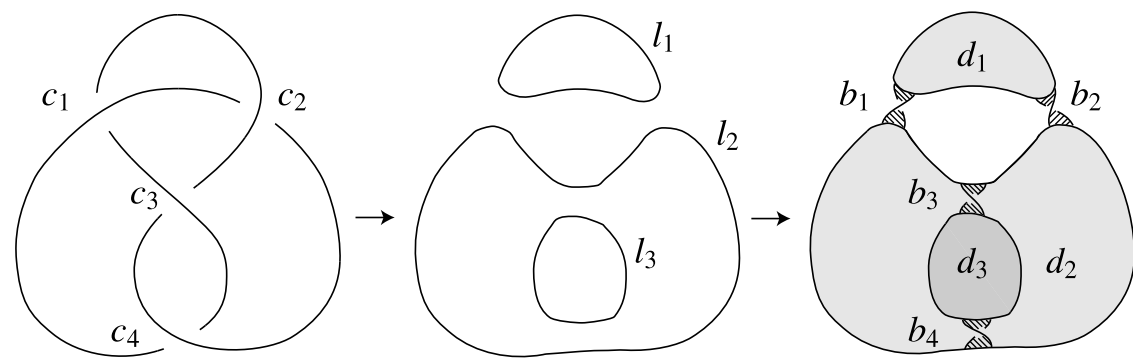

Diagram

State loops

$\sigma$-state surface

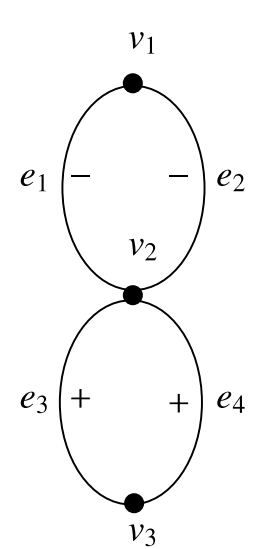

$\sigma$-state graph

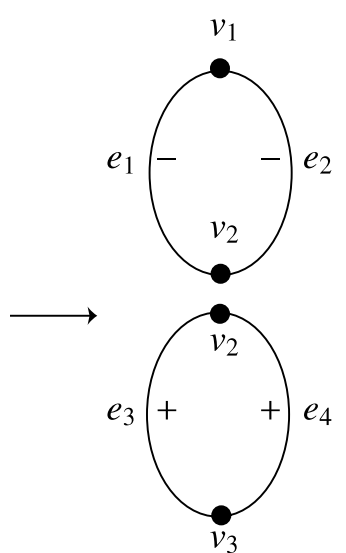

Blocks
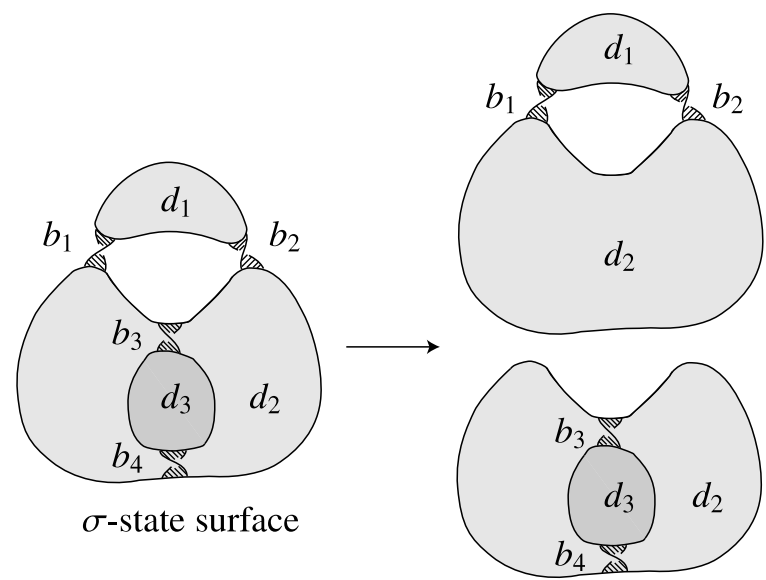

Figure 3. A state surface, $\sigma$-state graph and block decomposition, and Murasugi decomposition. 
ExAmple 2.6. We say that a diagram $D$ is homogeneous [4] if $D$ is $\vec{\sigma}$-homogeneous for the Seifert state $\vec{\sigma}$. Note that $D$ is automatically $\vec{\sigma}$-adequate since the $\vec{\sigma}$-state surface $F_{\vec{\sigma}}$ is orientable and thus $G_{\vec{\sigma}}$ has no loop.

We say that a diagram $D$ is semiadequate [16] if $D$ is $\sigma$-adequate for the positive state $\sigma_{+}$or the negative state $\sigma_{-}$. Note that $D$ is automatically $\sigma_{ \pm}$-homogeneous since $\sigma_{ \pm}\left(c_{j}\right)= \pm$ for all $j$.

We say that a diagram $D$ is adequate [29] if $D$ is $\sigma$-adequate for both the positive state $\sigma_{+}$and the negative state $\sigma_{-}$. Note also that $D$ is automatically $\sigma_{ \pm}$-homogeneous since $\sigma_{ \pm}\left(c_{j}\right)= \pm$ for all $j$.

EXAMPLE 2.7. We say that an arborescent link $L$ is strictly arborescent if the absolute value of each weight is greater than 1. Note that there exist a diagram $D$ of $L$ and a state $\sigma$ such that $D$ is $\sigma$-adequate and $\sigma$-homogeneous. Indeed, a strictly arborescent link $L$ is the boundary of a $\sigma$-state surface that is a Murasugi sum of twisted annuli or Möbius bands with one or more full twists. See [3] or [12] for the definition and construction of surfaces for arborescent links.

We now review the definition of essential surfaces. Let $M$ be an orientable compact 3-manifold and let $F$ be a compact surface, but not a 2-sphere, properly embedded in $M$, possibly with boundary. Let $i$ denote the inclusion map $F \rightarrow M$. We say that $F$ is $\pi_{1}$-injective if the induced map

$$
i_{*}: \pi_{1}(F) \rightarrow \pi_{1}(M)
$$

is injective. We say that $F$ is $\partial-\pi_{1}$-injective if the induced map

$$
i_{*}: \pi_{1}(F, \partial F) \rightarrow \pi_{1}(M, \partial M)
$$

is injective for every choice of two base points in $\partial F$. A surface $F$ in $M$ is $\pi_{1}$-essential if $F$ is $\pi_{1}$-injective, $\partial$ - $\pi_{1}$-injective and not $\partial$-parallel in $M$.

A disk $D$, embedded in $M$, is a compressing disk for $F$ if $D \cap F=\partial D$ and $\partial D$ is an essential loop in $F$. A disk $D$, embedded in $M$, is a $\partial$-compressing disk for $F$ if $D \cap F \subset \partial D$ is an essential arc in $F$ and

$$
D \cap \partial M=\partial D-\operatorname{int}(\mathrm{D} \cap \mathrm{F}) .
$$

We say that $F$ is incompressible or $\partial$-incompressible if there exists no compressing disk or $\partial$-compressing disk respectively for $F$. A surface $F$ in $M$ is essential if $F$ is incompressible, $\partial$-incompressible and not $\partial$-parallel in $M$. We remark that a $\sigma$ state surface $F_{\sigma}$ is $\pi_{1}$-essential in $E(K)$ if and only if the interpolating surface $\left(F_{\sigma}\right)^{\sim}$ obtained from $F_{\sigma}$ is essential in $E(K)$.

The following theorem gives a sufficient condition for the state surface to be $\pi_{1}$ essential.

THEOREM 2.8. If a diagram is both $\sigma$-adequate and $\sigma$-homogeneous for some state $\sigma$, then the $\sigma$-state surface is $\pi_{1}$-essential.

If $F_{\sigma}$ is nonorientable and $\pi_{1}$-essential, then the interpolating surface $\left(F_{\sigma}\right)^{\sim}$ is connected and essential. Therefore, the knot satisfies the Neuwirth conjecture [21], 
which states that, for any nontrivial knot $K$, there exists a closed surface $S$ that contains $K$ and is such that $S \cap E(K)$ is connected and essential in $E(K)$.

Corollary 2.9. If a diagram is $\sigma$-adequate and $\sigma$-homogeneous for a state $\sigma \neq \vec{\sigma}$, then the knot satisfies the Neuwirth conjecture. In particular, all adequate knots satisfy the Neuwirth conjecture.

REMARK 2.10. It can be confirmed that every 10-crossing knot diagram in the Rolfsen knot table [27], except for $8_{19}, 10_{124}, 10_{128}, 10_{134}, 10_{139}$ and $10_{142}$, is $\sigma$-adequate and $\sigma$-homogeneous for a positive or negative state $\sigma$ distinct from the Seifert state $\vec{\sigma}$. Also, every 11-crossing knot diagram in the Hoste-Thistlethwaite knot table [14], except for $K 11_{n} 93, K 11_{n} 95, K 11_{n} 118, K 11_{n} 126, K 11_{n} 136, K 11_{n} 169, K 11_{n} 171$, $K 11_{n} 180$ and $K 11_{n} 181$, is $\sigma$-adequate and $\sigma$-homogeneous for a positive or negative state $\sigma$ distinct from the Seifert state $\vec{\sigma}$. Furthermore, it can be checked that $10_{134}$, $10_{142}, K 11_{n} 93, K 11_{n} 95, K 11_{n} 136, K 11_{n} 169, K 11_{n} 171, K 11_{n} 180$ and $K 11_{n} 181$ bound $\pi_{1}$-essential, nonorientable checkerboard surfaces. (It may be necessary to deform the diagram by the Reidemeister move of type III.)

Remark 2.11. Futer et al. [10] estimated the hyperbolic volume of adequate knots by using the guts of state surfaces.

REMARK 2.12. We can construct a spanning surface other than $F_{\sigma}$ from a state $\sigma$ by letting the loop $l_{i}$ bound a disk $d_{i}^{\prime}$ in $B_{+}$. Theorem 2.8 holds for all state surfaces obtained by such a method. Moreover, we can construct a branched surface as in [13] that consists of disks $d_{1}, \ldots, d_{m}$ in $B_{-}$and disks $d_{1}^{\prime}, \ldots, d_{m}^{\prime}$ in $B_{+}$bounded by $l_{1}, \ldots, l_{m}$ respectively, and half twisted bands $b_{1}, \ldots, b_{n}$.

Remark 2.13. Suppose that a diagram $D$ is $\sigma$-adequate and $\sigma$-homogeneous for a state $\sigma$. If $F_{\sigma}$ is orientable, then it is a minimal genus Seifert surface by [11, Theorem 2] or [4, Corollary 4.1].

On the other hand, as pointed out by M. Hirasawa, a similar phenomenon need not occur in the nonorientable case. Indeed, there exist 2-bridge links with two continued fractions -3 2-2 3 and 23 2, where the notation follows Adams [1].

ReMark 2.14. The converse of Theorem 2.8 does not hold in general. It is true that if a $\sigma$-state surface $F_{\sigma}$ is $\pi_{1}$-essential, then the diagram $D$ is $\sigma$-adequate. However, it is not true in general that if a $\sigma$-state surface $F_{\sigma}$ is $\pi_{1}$-essential, then the diagram $D$ is $\sigma$-homogeneous.

By using a $\pi_{1}$-essential state surface, we can prove the next theorem, which tells us that we can deduce the triviality and splittability of a knot or link from its diagram. In this paper, we say that a diagram $D$ is nontrivial if it contains at least one crossing and that $D$ is nonsplit if it is connected.

THeorem 2.15. Let $K$ be a knot or link that admits a $\sigma$-adequate and $\sigma$-homogeneous diagram $D$ without nugatory crossings for some state $\sigma$. Then the following hold. 


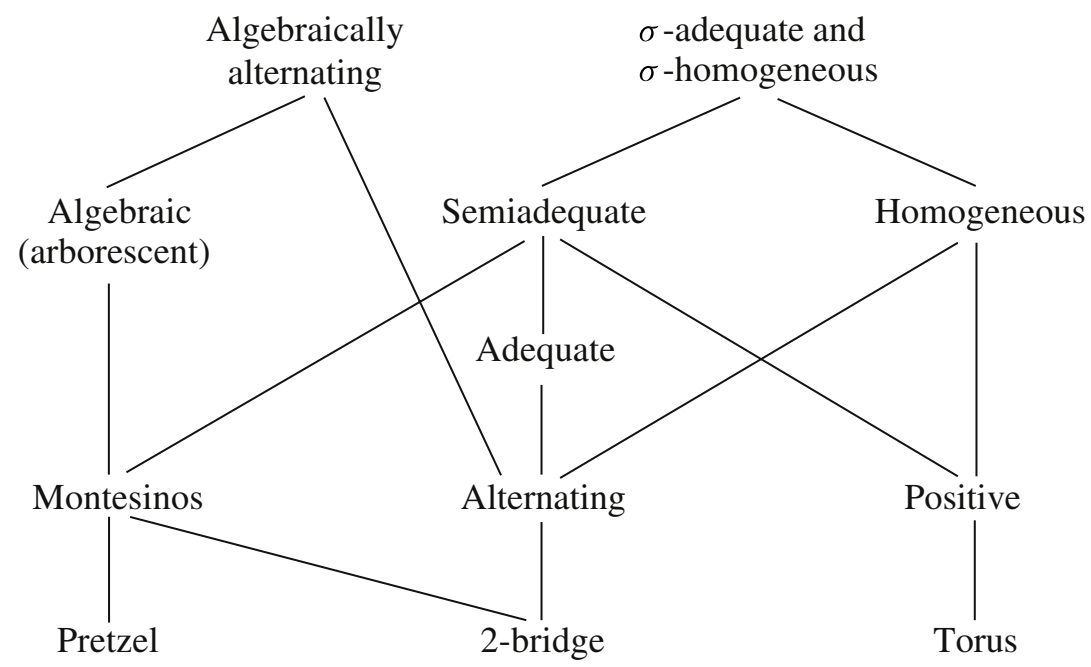

FIGURE 4. The Hasse diagram for the set of knot diagrams partially ordered by inclusion.

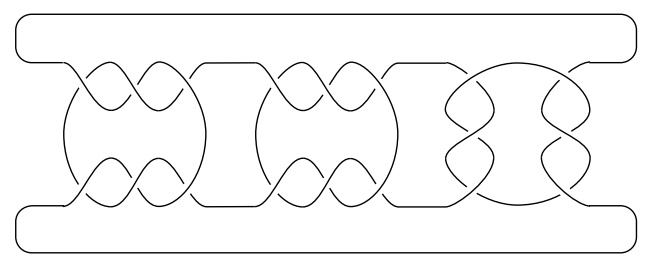

FigurE 5. An algebraic link that is neither $\sigma$-adequate nor $\sigma$-homogeneous for any state $\sigma$.

(1) $D$ is nontrivial if and only if $K$ is nontrivial.

(2) $D$ is nonsplit if and only if $K$ is nonsplit.

The determining problem for the triviality and splittability of a knot or link has been solved for the following classes. For triviality, the determining problem has been solved for alternating knots [19], homogeneous links [4], semiadequate links [29] and Montesinos knots [16]. For splittability, the determining problem has been solved for alternating links [18], homogeneous links [4], semiadequate links [29] and positive links [22].

Figure 4 shows the Hasse diagram of the various classes of knots and links. Here, almost all algebraic links have $\sigma$-adequate and $\sigma$-homogeneous diagrams for some state $\sigma$ (see Example 2.7), but some algebraic links seem to be neither $\sigma$-adequate nor $\sigma$-homogeneous for any state $\sigma$ (see Figure 5). Algebraically alternating knots and links are defined in [24] so that these classes include both alternating and algebraic knots and links. Some results on closed, incompressible surfaces are obtained in [24]. 


\section{Lemmas}

The next lemma is stated for knots, but it also holds for links $K$, provided that $E(K)-F$ is irreducible. Note that, for a connected diagram $D$ and a state surface $F_{\sigma}$, $E(K)-F_{\sigma}$ will be a handlebody and hence irreducible.

Lemma 3.1 [23, Lemma 2]. Let $K$ be a knot in $S^{3}$ and let $F$ be an incompressible, orientable surface properly embedded in $E(K)$. If $F$ is $\partial$-compressible in $E(K)$, then $F$ is a $\partial$-parallel annulus.

Similarly, we have the following lemmas.

Lemma 3.2 [25, Lemma 2.2]. Let $K$ be a knot in $S^{3}$ and $F$ be a $\pi_{1}$-injective nonorientable surface properly embedded in $E(K)$. If $F$ is not $\partial-\pi_{1}$-injective, then $F$ is an unknotted, half-twisted Möbius band and $K$ is trivial.

Lemma 3.3 [2, Theorem 9.8], [20, Proposition 2.3], [23, Theorem 2, 3]. Let $D$ be a reduced, prime, alternating diagram. Then the checkerboard surface obtained from $D$ is $\pi_{1}$-essential.

Let $F$ be a spanning surface for a link $K$. Suppose that there exists a 2-sphere $S$ that decomposes $S^{3}$ into two 3-balls $B_{1}$ and $B_{2}$ such that $F \cap S$ is a disk. Put $F_{i}=F \cap B_{i}$ for $i=1,2$. Then we say that $F$ has a Murasugi decomposition into $F_{1}$ and $F_{2}$, which we denote by $F=F_{1} * F_{2}$. Conversely, we say that $F$ is obtained from $F_{1}$ and $F_{2}$ by a Murasugi sum along a disk $F \cap S$.

Put $E=S-\operatorname{int}(F \cap S)$ and let $\delta$ be a disk in $B_{1}$ such that

$$
\delta \cap\left(F_{1} \cup E\right)=\partial \delta \cap\left(F_{1} \cup E\right)=\partial \delta
$$

and $\partial \delta \cap E$ consists of mutually disjoint $\operatorname{arcs} \alpha_{1}, \ldots, \alpha_{n}$. Then there exist mutually disjoint arcs $\alpha_{1}^{\prime}, \ldots, \alpha_{n}^{\prime}$ in $F \cap S$ that form the mutually disjoint loops given by $\alpha_{1} \cup \alpha_{1}^{\prime}, \ldots, \alpha_{n} \cup \alpha_{n}^{\prime}$ in $S$. Moreover, there exist mutually disjoint disks $\delta_{1}^{\prime}, \ldots, \delta_{n}^{\prime}$ in $B_{2}$ that are bounded by $\alpha_{1} \cup \alpha_{1}^{\prime}, \ldots, \alpha_{n} \cup \alpha_{n}^{\prime}$ respectively.

We call the disk $\delta \cup\left(\delta_{1}^{\prime} \cup \cdots \cup \delta_{n}^{\prime}\right)$ the extended disk of $\delta$ towards $B_{2}$. We remark that the extended disk of $\delta$ is uniquely determined by $\delta$ and that in general it intersects the interior of $F_{2}$.

The following key lemma extends [11, Theorem 1] to nonorientable surfaces.

Lemma 3.4. If $F_{1}$ and $F_{2}$ are $\pi_{1}$-essential, then $F=F_{1} * F_{2}$ is also $\pi_{1}$-essential.

Proof. Suppose that $F_{1}$ and $F_{2}$ are $\pi_{1}$-essential. We will show that the interpolating surface $(F)^{\sim}=F \tilde{\times} \partial I$ is essential. By [23, Claim 9], $(F)^{\sim},\left(F_{1}\right)^{\sim}$ and $\left(F_{2}\right)^{\sim}$ are incompressible and $\partial$-incompressible in $F \tilde{\times} I, F_{1} \tilde{\times} I$ and $F_{2} \tilde{\times} I$ respectively.

Suppose that $(F)^{\sim}$ is compressible. Let $C$ be a compressing disk for $(F)^{\sim}$ in the outside of $F \tilde{\times} I$. Put $E=S-\operatorname{int}(F \cap S)$. Without loss of generality, we may assume that $C$ and $E$ are in general position, and that the number of components of $C \cap E$ is minimal over all compressing disks $C$. 

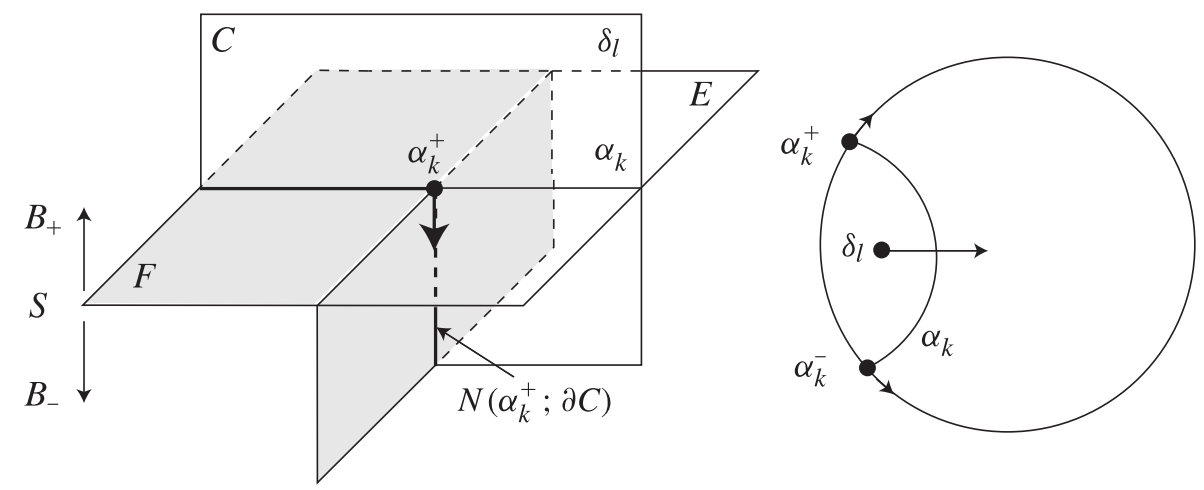

FIGURE 6. Marking $a_{k}^{ \pm}$with an arrow and an orientation induced by $\alpha_{k}$.

If $C \cap E=\emptyset$, then $C$ is a compressing disk for $\left(F_{1}\right)^{\sim}$ or $\left(F_{2}\right)^{\sim}$. Otherwise, $C \cap E$ consists of arcs, say $\alpha_{1}, \ldots, \alpha_{p}$. Let $\delta_{1}, \ldots, \delta_{q}$ be subdisks on $C$, separated by $\alpha_{1} \cup \cdots \cup \alpha_{p}$. For each arc $\alpha_{k}$, put $\partial \alpha_{k}=a_{k}^{+} \cup a_{k}^{-}$. A subarc $N\left(a_{k}^{ \pm} ; \partial C\right)$ runs over the disk $F \cap S$ and $F-S$. Then we mark $a_{k}^{ \pm}$with an arrow so that it runs from $F \cap S$ to $F-S$ (see Figure 6).

Suppose now that $F$ is not $\pi_{1}$-essential. We derive a contradiction by constructing a graph $G$ that possesses an impossible property. The following claim is needed to establish some properties of $C \cap E$ that will be useful in our construction.

Claim. For an outermost arc $\alpha_{k}$ and the corresponding outermost disk $\delta_{l}$, both arrows at $a_{k}^{ \pm}$turn out from $\delta_{l}$ (as in the right-hand side of Figure 6).

To prove the claim, we may assume without loss of generality that $\delta_{l} \subset B_{1}$. First, suppose that both arrows at $a_{k}^{ \pm}$turn into $\delta_{l}$ (see Figure 7). There exists an arc $\alpha_{k}^{\prime}$ that connects $a_{k}^{+}$and $a_{k}^{-}$on $F \cap S$. Also, the loop $\alpha_{k} \cup \alpha_{k}^{\prime}$ bounds a disk $\delta_{l}^{\prime}$ in $B_{2}$. We may now deduce that the extended disk $\delta_{l} \cup \delta_{l}^{\prime}$ towards $B_{2}$ is a compressing disk for $\left(F_{1}\right)^{\sim}$, since we have assumed that the number of components of $C \cap E$ is minimal. This contradicts our assumption that $F_{1}$ is $\pi_{1}$-essential.

Next, suppose that one arrow at $a_{k}^{ \pm}$turns into $\delta_{l}$ and another turns out from $\delta_{l}$ (see Figure 8). Similarly, there exists an arc $\alpha_{k}^{\prime}$ that connects $a_{k}^{+}$and $a_{k}^{-}$on $F \cap S$, and the loop $\alpha_{k} \cup \alpha_{k}^{\prime}$ bounds a disk $\delta_{l}^{\prime}$ in $B_{2}$. Then the extended disk $\delta_{l} \cup \delta_{l}^{\prime}$ towards $B_{2}$ is a $\partial$-compressing disk for $\left(F_{1}\right)^{\sim}$ since we have assumed that the number of components of $C \cap E$ is minimal. In either case, we have a contradiction and our claim is established.

We construct our graph $G$ on $C$ as follows. We assign a vertex $v_{l}$ to each subdisk $\delta_{l}$, and connect two vertices by an edge $e_{k}$ if the two corresponding subdisks have a common arc $\alpha_{k}$ of $C \cap E$. Note that $G$ is a tree, since any arc $\alpha_{k}$ separates $\delta$. Since, by our first claim, both arrows at the boundary of an outermost arc turn out from the corresponding outermost disk, we can assign a natural orientation to the corresponding outermost edge. We call this orientation of the edge $e_{k}$ the orientation induced by $\alpha_{k}$ (see Figure 6). 


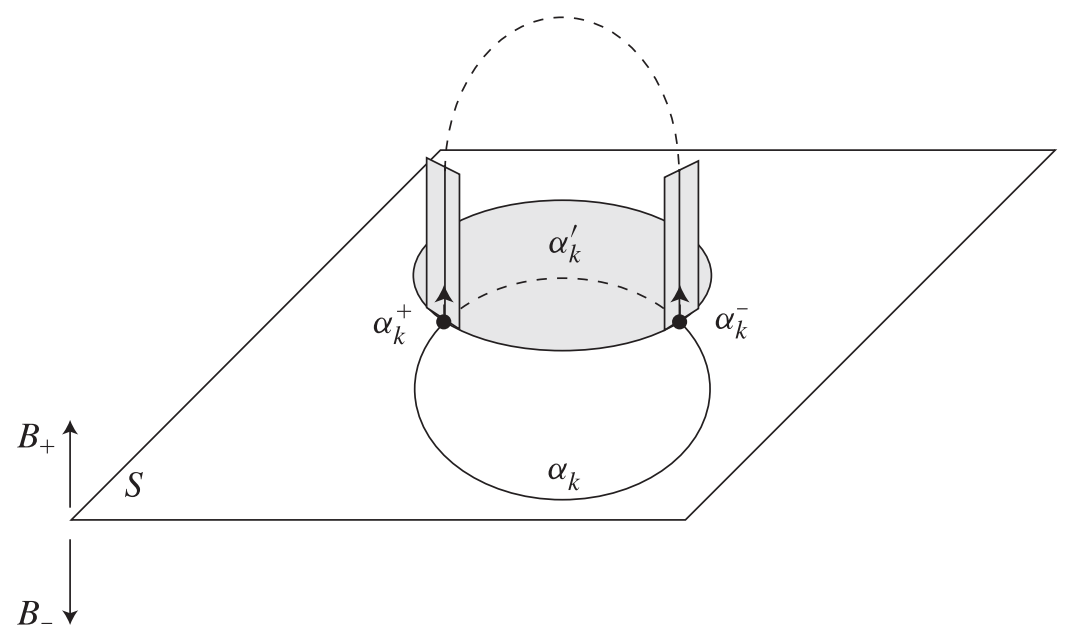

Figure 7. Both arrows at $a_{k}^{ \pm}$turn into $\delta_{l}$.

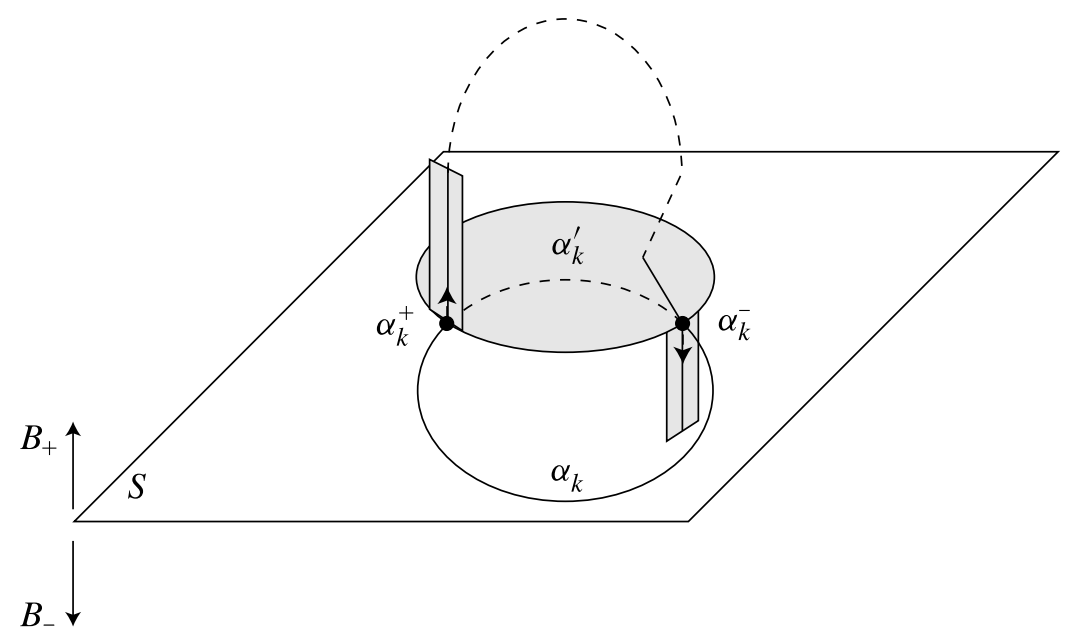

Figure 8 . One arrow at $a_{k}^{+}$turns into $\delta_{l}$ and another arrow at $a_{k}^{-}$turns out from $\delta_{l}$.

We say that a vertex of $G$ has depth $x$ if it becomes a vertex of degree one or zero after removing all vertices of depth less than $x$, where $x$ is a natural number. We define vertices corresponding to the outermost subdisks of $C$ as of depth 1. See Figure 9, where the depth of each vertex is indicated.

Our second claim will help us contradict our assumption that $(F)^{\sim}$ is compressible.

Claim. Every edge of $G$ has an induced orientation and every vertex has an edge oriented outwards. 

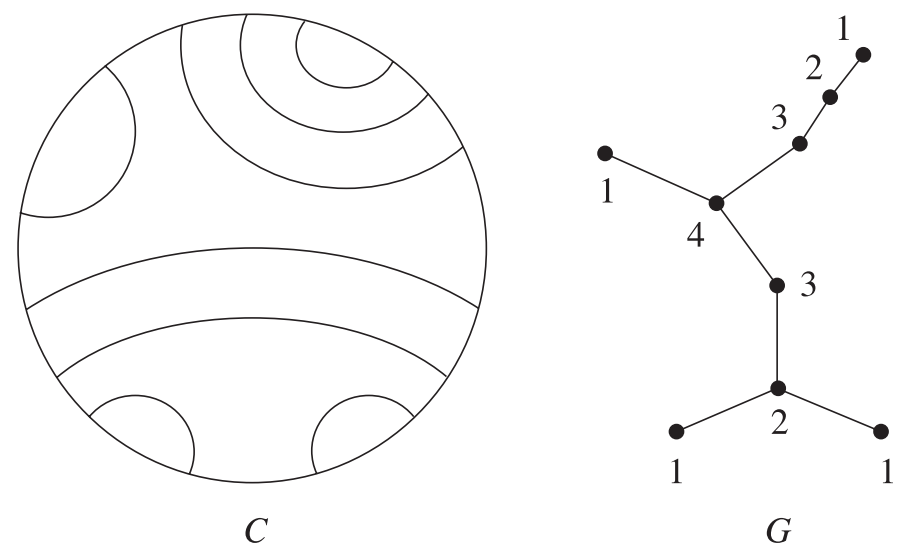

Figure 9. An example of $C \cap E$ on $C$ and the corresponding graph $G$.

We prove this claim by induction on the depth of $v_{l}$. If $v_{l}$ has depth 1 , then this is nothing but our previous claim.

Next, suppose that this claim holds for all vertices of depth less than $x$, and that $v_{l}$ has depth $x$. Let $N_{<x}\left(v_{l}\right)$ be the set of vertices that are adjacent to $v_{l}$ and have depth less than $x$. Since $G$ has no cycles, any vertex in $N_{<x}\left(v_{l}\right)$ has an edge oriented outwards to $v_{l}$. Without loss of generality, we may assume that $\delta_{l} \subset B_{1}$.

If $v_{l}$ becomes a degree-zero vertex after all vertices of depth less than $x$ are removed, then the extended disk $\delta_{l}^{\prime}$ of $\delta_{l}$ towards $B_{2}$ is a compressing disk for $\left(F_{1}\right)^{\sim}$. If $v_{l}$ becomes a degree-one vertex after all vertices of depth less than $x$ are removed, then let $e_{k}$ be the edge connecting $v_{l}$ to a vertex that is not contained in $N_{<x}\left(v_{l}\right)$, and let $\alpha_{k}$ be the corresponding arc. First, suppose that both arrows at $a_{k}^{ \pm}$turn into $\delta_{l}$. Then the extended disk $\delta_{l}^{\prime}$ of $\delta_{l}$ towards $B_{2}$ is a compressing disk for $\left(F_{1}\right)^{\sim}$. Next, suppose that one of the arrows at $a_{k}^{ \pm}$turns into $\delta_{l}$ and another turns out from $\delta_{l}$. Then the extended disk $\delta_{l}^{\prime}$ of $\delta_{l}$ towards $B_{2}$ is a $\partial$-compressing disk for $\left(F_{1}\right)^{\sim}$. In either case, we have a contradiction.

Hence $e_{k}$ has an orientation induced by $\alpha_{k}$, and $v_{l}$ has an edge oriented outwards. Our claim follows by induction.

This second claim leads us to a contradiction since $G$ is a tree. Hence $(F)^{\sim}$ is incompressible. It follows, by an elementary cutting-and-pasting argument, that $K$ is nonsplit. If $(F)^{\sim}$ is $\partial$-compressible, then it is $\partial$-parallel annulus by Lemma 3.1. Thus $F$ is not a $\partial-\pi_{1}$-injective Möbius band. Hence one of $F_{1}$ and $F_{2}$ is also not a $\partial-\pi_{1}$ injective Möbius band. This contradicts our assumption that both of $F_{1}$ and $F_{2}$ are $\pi_{1}$-essential. Therefore $F$ must be essential.

\section{Proofs}

Proof of Theorem 2.8. Suppose that a diagram $D$ is $\sigma$-adequate and $\sigma$-homogeneous for some state $\sigma$. Then the $\sigma$-state graph $G_{\sigma}$ may be decomposed into maximal blocks $G_{1}, \ldots, G_{n}$, each of which has no loop and has all of its edges of the same sign. 
Let $F_{1}, \ldots, F_{n}$ be the $\sigma$-state surfaces corresponding to $G_{1}, \ldots, G_{n}$. Then for each $i$, the boundary $\partial F_{i}$ represents an alternating diagram that is reduced and prime since $G_{i}$ has no loop and the block decomposition is maximal. By Lemma 3.3, $F_{i}$ is $\pi_{1}$-essential for each $i$. It follows by Lemma 3.4 that $F$ is also $\pi_{1}$-essential.

Proof of Theorem 2.15. Let $K$ be a knot or link that admits a $\sigma$-adequate and $\sigma$-homogeneous diagram $D$ without nugatory crossings. By Theorem 2.8, a $\sigma$-state surface $F_{\sigma}$ is $\pi_{1}$-essential.

Suppose first that $K$ is nontrivial. Then any diagram of $K$ has at least one crossing. Hence $D$ is nontrivial. Conversely, suppose that $D$ is nontrivial. Since $D$ has at least one crossing and does not have nugatory crossings, there exists a component of $F_{\sigma}$ that is not a disk. This shows that $K$ is nontrivial.

Suppose now that $K$ is nonsplit. Then any diagram of $K$ is connected. Hence $D$ is nonsplit. Conversely, suppose that $D$ is nonsplit. Since $D$ is connected, $F_{\sigma}$ is also connected. By a cutting-and-pasting argument on a splitting sphere, $K$ is nonsplit.

\section{Problems}

Here, we list four problems that we would like to solve in the future.

(1) Show that there exists a knot that has no $\sigma$-adequate and $\sigma$-homogeneous diagram. Furthermore, characterize the nature of knots and links that have $\sigma$-adequate and $\sigma$-homogeneous diagrams.

(2) Determine primeness, satelliteness, fibredness, smallness and tangle decomposability from a given $\sigma$-adequate and $\sigma$-homogeneous diagram.

(3) Show that, for a given knot, the number of all $\sigma$-adequate and $\sigma$-homogeneous diagrams without nugatory crossings is finite.

(4) Classify knots and links that have $\sigma$-adequate and $\sigma$-homogeneous diagrams.

We believe that essential state surfaces will be useful for solving these problems.

\section{Addendum}

Since our initial version of this paper, written in 2006, some progress has been made on related matters. We summarize some of those results here.

In [10, Theorem 3], Futer et al. used our main Theorem 2.8 to verify the Garoufalidis conjecture on a relation between the boundary slopes of a knot and its colored Jones polynomials.

In [6], Curtis and Taylor used an unpublished result of Adams and Kindred, which is based on the work here, to show that, for an alternating knot, the minimal integral boundary slope is given by the signature plus twice the minimum degree of the Jones polynomial. They also showed that the maximal integral boundary slope is given by the signature plus twice the maximum degree of the Jones polynomial. 


\section{Acknowledgements}

I thank Professor Kouki Taniyama for suggesting research on adequate knots and links using geometrical methods. Thanks are also due to Professor Jozef H. Przytycki for telling me about the positive or negative state and the Seifert state. Finally, I deeply appreciate the many efforts of the referees in reading this paper.

\section{References}

[1] C. Adams, The Knot Book: An Elementary Introduction to the Mathematical Theory of Knots (American Mathematical Society, Providence, RI, 2004), Revised reprint of the 1994 original.

[2] R. J. Aumann, 'Asphericity of alternating knots', Ann. of Math. (2) 64 (1956), 374-392.

[3] F. Bonahon and L. Siebenmann, 'Geometric splittings of classical knots and the algebraic knots of Conway', in: New Geometric Splittings of Classical Knots, London Mathematical Society Lecture Note Series, 75 (ed. L. Siebenmann) (Cambridge University Press, Cambridge, 2003).

[4] P. R. Cromwell, 'Homogeneous links', J. Lond. Math. Soc. (2) 39 (1989), 535-552.

[5] P. R. Cromwell, Knots and Links (Cambridge University Press, Cambridge, 2004).

[6] C. L. Curtis and S. Taylor, 'The Jones polynomial and boundary slopes of alternating knots', arXiv:0910.4912.

[7] R. Diestel, Graph Theory, 3rd edn, Graduate Texts in Mathematics, 173 (Springer, Berlin, 2005).

[8] A. Elliott, 'State cycles, quasipositive modification, and constructing H-thick knots in Khovanov homology', arXiv:0901.4039.

[9] P. Frankl and L. Pontrjagin, 'Ein Knotensatz mit Anwendung auf die Dimensionstheorie', Math. Ann. 102 (1930), 785-789.

[10] D. Futer, E. Kalfagianni and J. S. Purcell, 'Slopes and colored Jones polynomials of adequate knots', Proc. Amer. Math. Soc. 139 (2011), 1889-1896.

[11] D. Gabai, 'The Murasugi sum is a natural geometric operation', Contemp. Math. 20 (1983), 131-143.

[12] D. Gabai, 'Genera of the arborescent links', Mem. Amer. Math. Soc. 59 (1986).

[13] A. Hatcher and W. Thurston, 'Incompressible surfaces in 2-bridge knot complements', Invent. Math. 79 (1985), 225-246.

[14] J. Hoste and M. Thistlethwaite, 'The Hoste-Thistlethwaite Table of 11 Crossing Knots', in http:// katlas.math.toronto.edu/wiki/The_Hoste-Thistlethwaite_Table_of_11_Crossing_Knots.

[15] L. H. Kauffman, 'State models and the Jones polynomial', Topology 26 (1987), 395-407.

[16] W. B. R. Lickorish and M. B. Thistlethwaite, 'Some links with nontrivial polynomials and their crossing numbers', Comment. Math. Helv. 63 (1988), 527-539.

[17] S. Matveev, Algorithmic Topology and Classification of 3-manifolds, 2nd edn, Algorithms and Computation in Mathematics, 9 (Springer, Berlin, 2007).

[18] W. Menasco, 'Closed incompressible surfaces in alternating knot and link complements', Topology 23 (1984), 37-44.

[19] W. Menasco and M. Thistlethwaite, 'A geometric proof that alternating knots are nontrivial', Math. Proc. Camb. Phil. Soc. 109 (1991), 425-431.

[20] W. Menasco and M. Thistlethwaite, 'The classification of alternating links', Ann. of Math. (2) 138 (1993), 113-171.

[21] L. Neuwirth, 'Interpolating manifolds for knots in $S^{3}$, Topology 2 (1964), 359-365.

[22] M. Ozawa, 'Closed incompressible surfaces in the complements of positive knots', Comment. Math. Helv. 77 (2002), 235-243.

[23] M. Ozawa, 'Nontriviality of generalized alternating knots', J. Knot Theory Ramifications 15 (2006), 351-360.

[24] M. Ozawa, 'Rational structure on algebraic tangles and closed incompressible surfaces in the complements of algebraically alternating knots and links', Topology Appl. 157 (2010), 1937-1948. 
[25] M. Ozawa and Y. Tsutsumi, 'Totally knotted Seifert surfaces with accidental peripherals', Proc. Amer. Math. Soc. 131 (2003), 3945-3954.

[26] M. D. Pabiniak, J. H. Przytycki and R. Sazdanovic, 'On the first group of the chromatic cohomology of graphs', Geom. Dedicata 140 (2009), 19-48.

[27] D. Rolfsen, Knots and Links (AMS Chelsea Publishing, Providence, RI, 2003).

[28] H. Seifert, 'Über das Geschlecht von Knoten', Math. Ann. 110 (1934), 571-592.

[29] M. B. Thistlethwaite, 'On the Kauffman polynomial of an adequate link', Invent. Math. 93 (1998), 285-296.

\section{MAKOTO OZAWA, Department of Natural Sciences, Faculty of Arts and Sciences, Komazawa University, 1-23-1 Komazawa, Setagaya-ku, Tokyo, 154-8525, Japan e-mail: w3c@komazawa-u.ac.jp}

